\title{
Minocycline Toxicity: Case Files of the University of Massachusetts Medical Toxicology Fellowship
}

\author{
Matthew D. Zuckerman • Katherine L. Boyle • \\ Christopher D. Rosenbaum
}

Published online: 10 July 2012

(C) American College of Medical Toxicology 2012

Keywords Minocycline $\cdot$ Tetracycline $\cdot$ Blue skin pigmentation $\cdot$ Black bone disease

\section{Case Presentation}

A 61-year-old man was brought to the emergency department (ED) for shortness of breath, fatigue, frequent falls, and bluish discoloration of his skin. The primary care physician transferred the patient due to concern for cyanosis. On presentation to the ED, the patient was oriented but appeared fatigued. The patient was afebrile and had the following vital signs: blood pressure $161 / 88 \mathrm{mmHg}$, pulse $71 / \mathrm{min}$, respiratory rate $16 / \mathrm{min}$, and oxygen saturation $100 \%$ on room air by fingertip pulse oximetry. On physical exam, pupils were $4 \mathrm{~mm}$ and reactive bilaterally, extraocular movements were intact, and there was no nystagmus. Anicteric sclerae were notable for blue pigmentation (Fig. 1). Heart sounds were regular, without murmurs, rubs, or gallops. Lung sounds were clear to auscultation bilaterally. Other than ataxia, the neurological exam was unremarkable. The skin had a generalized bluish tinge, especially on the arms, with darker pigmentation on the cheeks (Figs. 2, 3 and 4). There was acne on the back and facial rosacea. Bluish discoloration was also noted under the proximal nail beds (Fig. 5). When questioned about the skin discoloration, the patient and his family members described an insidious onset. Photographs of the patient from years ago confirmed that this was not congenital. Medical history included acne, orthostatic

M. D. Zuckerman $(\bowtie) \cdot$ K. L. Boyle $\cdot$ C. D. Rosenbaum Division of Medical Toxicology, Department of Emergency Medicine, University of Massachusetts Medical School, 55 Lake Avenue North,

Worcester, MA 01655, USA

e-mail: Matthew.Zuckerman@umassmemorial.org hypotension, and Parkinson's disease, for which a deep brain stimulator had been surgically implanted. A comprehensive medication list was not immediately available; however, the patient denied any recent medication additions or adjustments. The patient was unemployed, denied any recent travel, and no other members of his household were complaining of fatigue or similar blue skin discoloration.

\section{What is the Differential Diagnosis of these Skin Changes?}

When developing a differential diagnosis (see Table 1) for bluish skin discoloration, one must determine if the patient has cyanosis. A significant toxicologic cause of blue skin appearance from cyanosis is methemoglobinemia. A careful history may help differentiate between acquired versus congenital methemoglobinemia. Recent exposure to dapsone, benzocaine, lidocaine, nitrates, or aniline dye raises suspicion for acquired methemoglobinemia. Sulfhemoglobinemia should be considered in patients with a positive methemoglobin reading on co-oximetry, but who do not respond to methylene blue treatment. Oxidizing agents that may cause methemoglobinemia can also produce sulfhemoglobinemia in the presence of sulfur compounds, such as sulfonamide derivatives, hydrogen sulfide, or gastrointestinal sources [1].

Causes of noncyanotic skin discoloration are extensive. Metal deposition (e.g., silver, gold, bismuth) causes blue skin pigmentation. Chronic or improper exposure to silver can result in a silvery blue or gray skin discoloration known as argyria. Argyria is caused by silver deposition in the skin, specifically within fibroblasts, macrophages, and in the extracellular matrix. These skin changes are often most significant in sun-exposed areas [2]. Similarly, chrysiasis refers to 


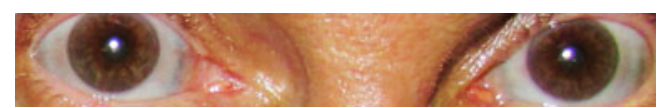

Fig. 1 Bluish pigmentation in the patient's eyes

gray-blue skin resulting from parenteral exposure to gold salts, classically used to treat rheumatoid arthritis. Like argyria, chrysiasis is most prominent in sun-exposed areas. Gold particles are deposited in a perivascular distribution throughout the dermis. Bismuth salts also cause a dark discoloration of the gingiva and tongue that can be mistaken for cyanosis. Although the pigmentation associated with metals is very different than the bluish discoloration associated with some antibiotics, a recent visual diagnosis feature in the New England Journal of Medicine indicated that almost one fifth of the respondents could not differentiate between the two [3]. Medications like amiodarone, doxorubicin, bleomycin, and phenothiazines have also been implicated in bluish skin discoloration [4].

Inborn errors of metabolism (e.g., alkaptonuria) may also discolor the skin. Alkaptonuria is a rare autosomal recessive disorder defined by increased levels of homogentisic acid (HGA) in the urine and connective tissues. Patients with alkaptonuria suffer from pigmentation changes called ochronosis. In the connective tissues, oxidation of HGA forms a blue-black pigment that is most visible in the sclerae and ears. Multiple case reports describe patients with toxicologic causes of skin discoloration misdiagnosed with alkaptonuria [5].

\section{What is the Appropriate Workup for these Skin Changes?}

As with any toxicologic exposure, a thorough exposure history is important. This should include medications, as well as herbal supplements, vitamins, over-the-counter

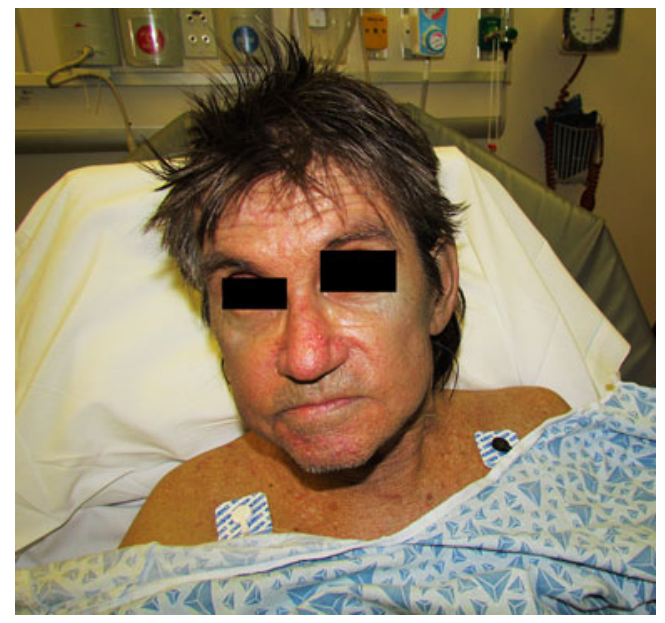

Fig. 2 Pigmentation of the face

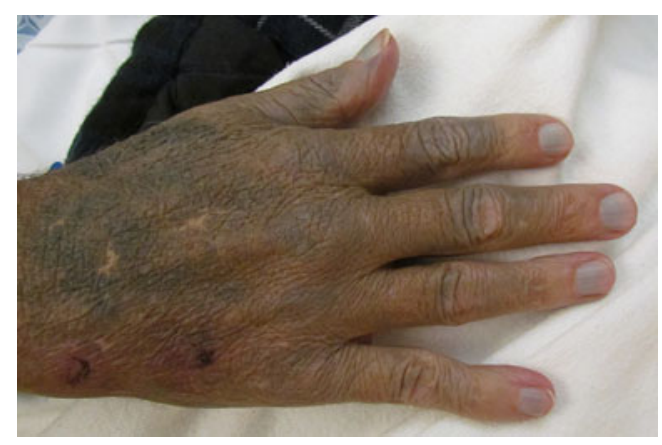

Fig. 3 Pigmentation of the hands

medications, possible surgical implants, and environmental and occupational exposures.

Evaluation of renal and hepatic function should be obtained in order to identify deficiencies in metabolism resulting in increased concentrations of toxic agents. Evaluation for hemolysis, vitamin $\mathrm{B}_{12}$ deficiency, and compensatory polycythemia should be considered, especially with chronic cyanosis. Differentiating between hematologic and soft tissue causes of discoloration may be obvious based on gross visualization; however, blood sampling and biopsy of tissue sections may be helpful.

Direct testing for abnormal forms of hemoglobin (i.e., methemoglobin, sulfhemoglobin) may be performed on venous or arterial blood. More recently, noninvasive cooximeters have gained popularity, although the reliability of such co-oximeters can vary [6]. If this testing is not available, then rapid delineation of hematologic versus soft tissue sources of discoloration may be suggested by gross appearance of blood samples and by blood gas analysis (see Fig. 6). Methemoglobinemia causes blood darkening by altering the absorption of light, while bluish skin discoloration associated with argyria and tetracycline toxicity has no effect on blood coloration. Blood gas analysis is useful for determining if there is dissociation between the measured partial pressure of oxygen and the predicted oxygen saturation, commonly found in congenital and acquired hemoglobinopathies. If the blood appears cyanotic to the naked eye with a low pulse oximetry reading and correspondingly low partial pressure of oxygen, then the discoloration is likely due to the presence of elevated levels of deoxyhemoglobin. In these cases, a cause of the hypoxemia must be investigated (e.g., pulmonary fibrosis associated with amiodarone toxicity, cardiac shunt, etc.). Bright-red arterial blood coloration with normal oxygen saturation and appropriate partial

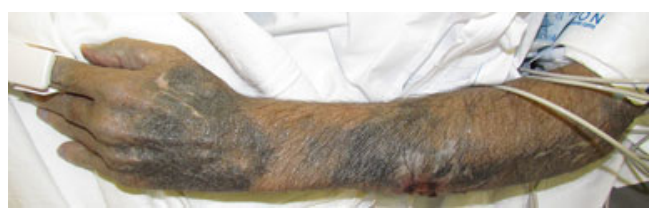

Fig. 4 Pigmentation of the arms 


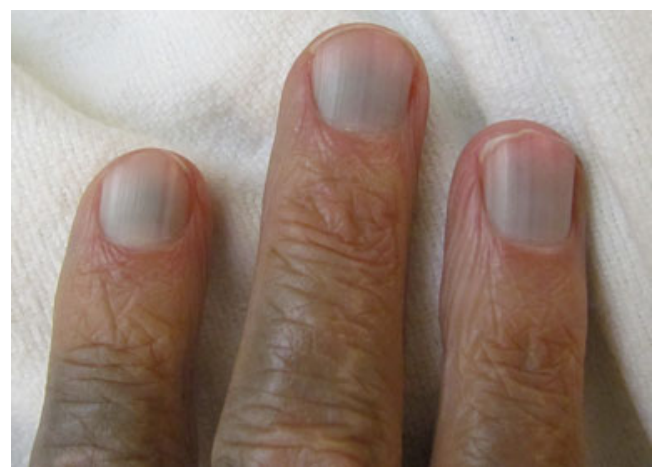

Fig. 5 Bluish pigmentation of the patient's nail beds

pressure of oxygen (often elevated due to the supplemental oxygen these patients are usually administered) indicates a discoloration independent of blood spectral absorption and oxygen content, likely a result of soft tissue or bone discoloration, which is discussed further below. Dark arterial blood (grossly similar to a venous blood sample) with low oxygen saturation via oximetry and normal or elevated partial pressure of oxygen indicates a possible hemoglobinopathy. Additionally, the hyperoxia test may be helpful: administration of $100 \%$ oxygen via face mask will typically increase oxygen saturation in patients with cardiopulmonary

Table 1 Causes of blue discoloration

Genetic
Alkaptonuria
Congenital methemoglobinemia
Acquired
Toxins
Arsine gas
Metals
Silver (argyria)
Copper
Gold (chrysiasis)
Bismuth (bismuthia)
Dyes
Methylene blue
Aniline
Drugs
Amiodarone
Chlorpromazine
Phenacetin
Minocycline
Oxidative stress resulting in methemoglobinemia, such as from
Nitrites
Dapsone
Local anesthetics (e.g. benzocaine)
Phenazopyridine

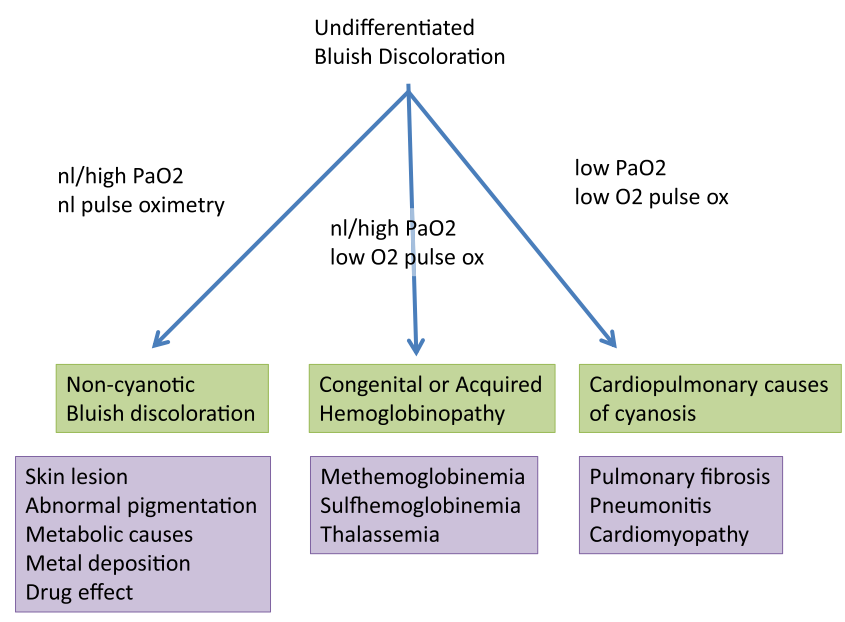

Fig. 6 Differentiating types of blue skin discoloration via $\mathrm{PaO}_{2}$ and oxygen saturation by pulse oximetry

disease (in absence of shunt) but will not have any effect on hemoglobinopathies or tissue pigmentation.

Skin biopsy of affected areas may be evaluated with light microscopy and Perls' stain (indicating iron) as well as Fontana-Masson stain (indicating the presence of melanin). Electron microscopy can also demonstrate pigment deposition extracellularly or intracellularly and free pigment vs. membrane-bound pigment, although this is not usually required. Direct examination of underlying bony structures and organs has been helpful in understanding the source of some discoloration but is not routinely required. For example, palate mucosal discoloration may actually be due to underlying "black bone disease" of the deeper bony structure.

Radiographs of the chest, or of areas of discoloration, may be helpful in diagnosing advanced lung fibrosis, cardiomegaly associated with cardiomyopathy, or in some cases deposition of radio-opaque metals or drug metabolites.

Checking levels of causative agents may be helpful (e.g., heavy metals), but these tests are generally not available in a timely manner. Additionally, the cumulative exposure often has better correlation with skin discoloration side effects than would a given random blood level. Thus, a history of prolonged exposure might give more understanding of what blood levels had been before presentation.

\section{Case Continuation}

The patient's normal serum chemistry and liver function tests ruled out renal or hepatic failure impairing medication elimination. The patient's bluish discoloration was not diffuse nor was the shade of blue consistent throughout. Along with normal oxygen saturation by pulse oximetry, this skin discoloration pattern made concerns for cyanosis and hemoglobinopathies less likely. The patient's ECG and chest X-ray did not 
demonstrate any acute cardiopulmonary disease. Hemoglobin concentration of $10.7 \mathrm{~g} / \mathrm{dL}$ (MCV $90 \mathrm{fL}$ ) was consistent with a normocytic anemia.

A medical history revealed that the patient had a long history of acne treated with minocycline $100 \mathrm{mg}$ orally twice daily for several years, and his Parkinson's disease was treated for several years with carbidopa/levodopa. Based on clinical presentation and medical history, it was felt that the patient's skin changes were caused by long-term minocycline use.

\section{What is the Clinical Spectrum of Tetracycline-induced Skin Changes?}

Minocycline is a synthetic tetracycline derivative with rapid absorption and prolonged half-life $\left(t^{1} / 2=15.5 \mathrm{~h}\right)$. It is more lipophilic than older tetratcyclines, resulting in rapid tissue distribution $[7,8]$. Because of its rapid absorption and long half-life, minocycline is commonly used as an adjunctive treatment for acne and rosacea [9]. Since its initial use in the 1960s, there have been reports of discoloration of various body tissues in both animals and humans. Unfortunately, the insidious onset of pigmentation delays patient selfrecognition of the problem, and the variability in patterns of pigmentation encumbers physician recognition [10]. Theories explaining patterns of pigmentation include drug complexes with iron, enhancement of melanocytes, and direct oxidation of drug resulting in pigmented metabolites [11]. Although opinions vary regarding the precise cause of minocycline-related skin pigmentation, there is general agreement about the categories of identified tissue discoloration.

Cutaneous blue pigmentation often limited to areas of inflammation, acne, or scarring is known as type I pigmentation [11]. This is often found on the face and acne of the back, although any area of inflammation is at risk, including areas of recent skin trauma related to intravenous catheters and procedures, psoriasis, eczema, etc. $[12,13]$. This pattern may occur sooner than other types and is independent of cumulative dose. Light and electron microscopy-analyzed tissue sections demonstrate pigment-laden granules within perivascular macrophages [12]. These pigments stain positively with Perls' stain, indicating the presence of iron and supporting the hypothesis that the granules contain minocycline metabolites complexed with iron $[11,14]$.

Type II pigmentation typically involves blue-black or gray pigmentation of otherwise normal skin on the shins, ankles and arms. Increasing cumulative dose is a risk factor for this type of discoloration, often involving collective doses of more than $100 \mathrm{~g}$ [15]. Tissue sections of skin stain positive for Perls' stain (indicating iron) as well as Fontana-
Masson stain (indicating the presence of melanin) [10, 15]. Electron microscopy of these same samples demonstrates membrane-bound pigment collections.

Type III pigmentation is similar to hyperpigmentation and presents as generalized brown coloration, especially in sun-exposed areas. Complexes of melanin and minocycline-melanin complex are theorized to cause the pigment and melanization of the basal layer [16]. Biopsy of a patient's sun-exposed neck demonstrated membrane-bound pigment in dermal macrophages as well as pigment deposition in extracellular collagen bundles [17].

Thus, it appears that type I staining occurs after a short exposure period with low cumulative dose (often less than $10 \mathrm{~g}$ ). Types II and III staining occur after months of treatment with a larger cumulative dose (100 g or more). Additionally, patients receiving minocycline for rosacea appear to be more susceptible to pigmentation than those receiving it for acne ( 15 vs. $3 \%$ of those on long-term therapy) $[11,18]$.

Other types of pigmentation (each separate entity at various points referred to as type IV) have been described as either blue-gray discoloration of scarring on the back (histologically unique from type I) or bluish pigmentation of the lips $[10,19]$.

The bluish discoloration of acne on our patient's back and face was consistent with type I staining. The dark blue staining of his arms and hands is more consistent with type II staining. Pigmentation was not limited to skin and also involved fingernail beds and sclera.

\section{Are there other Manifestations of Minocycline Discoloration?}

The oral cavity is a common place for discoloration involving both the teeth and the mucosa [20]. This may be due to salivary excretion of the lipophilic minocycline; however, this does not explain why dental staining is characteristically found on the middle third of teeth while saliva collects near the gum line [21]. A variety of theories for minocycline staining of teeth, include (1) extrinsic etching of enamel by high concentrations of drug, (2) intrinsic absorption from plasma with darkening due to oxidation, and (3) metabolism of minocycline to hemosiderin with resultant iron binding and formation of insoluble complexes [22]. Discoloration of oral mucus membranes may be related to melanization of basal cell layers (similar to type III skin reactions); however, not all oral pigmentation is due to change in the mucosal layer. In some patients, the membrane biopsy is normal, and pigmentation arises from deeper bones found to have a blue/black 
discoloration referred to as black bone disease [23]. Minocycline pigmentation limited to the tongue has been reported [24].

Minocycline also causes discoloration of other body tissues. Canine studies demonstrate discolored thyroid tissue $[25,26]$. Discoloration was less if animals simultaneously received thyroid supplementation, leading to the theory that thyroid activity and oxidation of absorbed minocycline by the thyroid peroxidases lead to pigment deposition [27]. These findings have been reproduced in human case reports. Glandular deposition of iron-bound pigment may be responsible for several cases of black galactorrhea in women [28, 29]. It is unclear if similar deposits cause discoloration of connective and cardiac valvular tissues [30].

Discoloration of the nail bed, sclera, and face has also been described, as well as bluish "conjunctival cysts" that appear like blue subconjunctival hemorrhages [13, 17, 31-33]. Some of this discoloration may occur after weeks or longer.

Black bone disease manifests in the skull, pelvis, shoulder, vertebrae, and costal cartilage $[34,35]$. Biopsies of discolored bone may fluoresce under UV light, similar to other kinds of tetracycline bone deposition; however, other studies do not find fluorescence. These conflicting findings may result from variations in tissue fixatives and preparation.

\section{What is the Treatment and Prognosis for Minocycline-induced Skin Discoloration?}

The treatment for minocycline-induced skin pigmentation primarily involves cessation of minocycline therapy. While case reports describe spontaneous resolution of discoloration ranging from weeks to months, some people experience irreversible effects. Interventions, such as Q-laser therapy, may be of benefit in certain cases [36-38].

\section{Case Continuation}

The emergency department physician initially evaluating the patient consulted the toxicology service for assistance in determining the cause of the patient's apparent cyanosis and whether this was related to his other complaints. The consultants felt the patient's bluish discoloration most likely related to his long-term minocycline use, and that it appeared unrelated to his presenting complaints. The patient was hospitalized for further evaluation of his weakness and dyspnea. It was ultimately felt that the patient was dehydrated, which had exacerbated the orthostatic hypotension associated with his Parkinson's disease. Fluid hydration lead to clinical improvement in the patient's symptoms, although the skin and eye discoloration did not change during hospitalization. As the patient's morbidity from minocycline was limited to cosmetic effects, no intervention was undertaken while he was hospitalized. His minocycline was discontinued, and he was instructed to follow up with a dermatologist regarding possible laser therapy on an outpatient basis.

Conflicts of Interest None

\section{References}

1. Fernandez-Frackelton M, Bocock J (2010) Chapter 29: Cyanosis. In: Marx JA, Hockberger RS, Walls RM, Adams J, Rosen P (eds) Rosen's emergency medicine: concepts and clinical practice. Elsevier, Mosby, pp 211-216

2. Becker M, Ewald M (2011) Chapter 99: Silver. In: Nelson L, Lewin N, Howland M, Goldfrank L, Flomenbaum N (eds) Goldfrank's toxicologic emergencies. McGraw-Hill Professional, New York, pp 1321-1325

3. Johnston AM, Memon AA (1999) Mystery of the blue pigmentation. N Engl J Med 340(20):1597-1598

4. Nelson LS, Lewin NA (2011) Chapter 29: Dermatologic principles. In: Nelson L, Lewin N, Howland M, Goldfrank L, Flomenbaum N (eds) Goldfrank's toxicologic emergencies. McGraw-Hill Professional, p 410-422

5. Suwannarat P, Phornphutkul C, Bernardini I, Turner M, Gahl WA (2004) Minocycline-induced hyperpigmentation masquerading as alkaptonuria in individuals with joint pain. Arthritis Rheum 50 (11):3698-3701

6. Feiner JR, Bickler PE, Mannheimer PD (2010) Accuracy of methemoglobin detection by pulse $\mathrm{CO}$-oximetry during hypoxia. Anesth Analg 111(1):143-148

7. Kelly RG, Kanegis LA (1967) Metabolism and tissue distribution of radioisotopically labelled minocycline. Toxicol Appl Pharmacol 11(1):171-183

8. Agwuh KN, MacGowan A (2006) Pharmacokinetics and pharmacodynamics of the tetracyclines including glycylcyclines. J Antimicrob Chemother 58(2):256-265

9. Gallo R, Drago F, Paolino S, Parodi A (2010) Rosacea treatments: what's new and what's on the horizon? Am J Clin Dermatol 11 (5):299-303

10. Mouton RW, Jordaan HF, Schneider JW (2004) A new type of minocycline-induced cutaneous hyperpigmentation. Clin Exp Dermatol 29(1):8-14

11. Eisen D, Hakim MD (1998) Minocycline-induced pigmentation. Incidence, prevention and management. Drug Saf 18(6):431-440

12. Fenske NA, Millns JL, Greer KE (1980) Minocycline-induced pigmentation at sites of cutaneous inflammation. JAMA 244 (10):1103-1106

13. Richter JC, Lyons B (2010) Medical image. Old blue eyes. Prolonged therapy with minocycline. N Z Med J 123(1318):79-80

14. Bowen AR, McCalmont TH (2007) The histopathology of subcutaneous minocycline pigmentation. J Am Acad Dermatol 57 (5):836-839

15. Argenyi ZB, Finelli L, Bergfeld WF, Tuthill RJ, McMahon JT, Ratz JL et al (1987) Minocycline-related cutaneous hyperpigmentation as demonstrated by light microscopy, electron microscopy and X-ray energy spectroscopy. J Cutan Pathol 14(3):176-180

16. Simons JJ, Morales A (1980) Minocycline and generalized cutaneous pigmentation. J Am Acad Dermatol 3(3):244-247 
17. Sabroe RA, Archer CB, Harlow D, Bradfield JW, Peachey RD (1996) Minocycline-induced discolouration of the sclerae. Br J Dermatol 135(2):314-316

18. Dwyer CM, Cuddihy AM, Kerr RE, Chapman RS, Allam BF (1993) Skin pigmentation due to minocycline treatment of facial dermatoses. Br J Dermatol 129(2):158-162

19. Chu P, Van SL, Yen TSS, Berger TG (1994) Minocycline hyperpigmentation localized to the lips: an unusual fixed drug reaction? J Am Acad Dermatol 30(5 Pt 1):802-803

20. LaPorta VN, Nikitakis NG, Sindler AJ, Reynolds MA (2005) Minocycline-associated intra-oral soft-tissue pigmentation: clinicopathologic correlations and review. J Clin Periodontol 32(2):119-122

21. Rosen T, Hoffmann TJ (1989) Minocycline-induced discoloration of the permanent teeth. J Am Acad Dermatol 21(3 Pt 1):569

22. Cheek CC, Heymann HO (1999) Dental and oral discolorations associated with minocycline and other tetracycline analogs. J Esthet Dent 11(1):43-48

23. Odell EW, Hodgson RP, Haskell R (1995) Oral presentation of minocycline-induced black bone disease. Oral Surg Oral Med Oral Pathol Oral Radiol Endod 79(4):459-461

24. Meyerson MA, Cohen PR, Hymes SR (1995) Lingual hyperpigmentation associated with minocycline therapy. Oral Surg Oral Med Oral Pathol Oral Radiol Endod 79(2):180-184

25. Azuma N, Hashimoto N, Nishioka A, Sano H (2010) Black thyroid. Intern Med 49(16):1835-1836

26. Benitz KF, Roberts GK, Yusa A (1967) Morphologic effects of minocycline in laboratory animals. Toxicol Appl Pharmacol 11 (1):150-170
27. Billano RA, Ward WQ, Little WP (1983) Minocycline and black thyroid. JAMA 249(14):1887

28. Basler RS, Lynch PJ (1985) Black galactorrhea as a consequence of minocycline and phenothiazine therapy. Arch Dermatol 121 (3):417-418

29. Hunt MJ, Salisbury EL, Grace J, Armati R (1996) Black breast milk due to minocycline therapy. Br J Dermatol 134(5):943-944

30. Belcher E, Soni M, Azeem F, Sheppard MN, Petrou M (2009) Minocycline-induced pigmentation of the aortic valve and sinuses of Valsalva. Ann Thorac Surg 88(5):1704

31. Ban M, Kitajima Y (2007) Nail discoloration occurring after 8 weeks of minocycline therapy. J Dermatol 34(10):699-701

32. Tavares J, Leung WWS (2011) Discoloration of nail beds and skin from minocycline. CMAJ 183(2):224

33. Bayne-Poorman M, Shubrook J (2010) Bluish pigmentation of face and sclera. J Fam Pract 59(9):519-522

34. Pandit S, Hadden W (2004) Black pigmentation of bone due to long-term minocycline use. Surgeon 2(4):236-237

35. Dummett C, Barens G (1967) Pigmentation of the oral tissues: a review of the literature. J Periodontol 38(5):369-378

36. Green D, Friedman KJ (2001) Treatment of minocycline-induced cutaneous pigmentation with the Q-switched Alexandrite laser and a review of the literature. J Am Acad Dermatol 44(2 Suppl):342-347

37. Bogdan Allemann I, Kaufman J (2010) Fractional photothermolysis - an update. Lasers Med Sci 25(1):137-144

38. Tierney EP, Kouba DJ, Hanke CW (2009) Review of fractional photothermolysis: treatment indications and efficacy. Dermatol Surg 35(10):1445-1461 